\title{
NLTE Abundances from Magellanic Cloud B Stars
}

\author{
Andreas J. Korn and Bernhard Wolf \\ Landessternwarte Königstuhl, 69117 Heidelberg, Germany
}

\begin{abstract}
Nine MC young globular cluster and field stars are analysed using fully line-blanketed LTE ATlas 9 atmospheres and the Detail/ SURFACE non-LTE line formation code. CNO mixing seems to occur much earlier than predicted by standard theory.
\end{abstract}

\section{Observations}

Spectra were taken with CASPEC $(R=20000)$ on the ESO $3.6 \mathrm{~m}$ telescope at La Silla/Chile achieving a $\mathrm{S} / \mathrm{N}$ of around 100. The stars show no $\mathrm{H} \alpha$ emission and are slow rotators with $v \sin i<65 \mathrm{~km} / \mathrm{s}$ ( $c f$. Reitermann et al. 1990).

\section{Model Atmosphere Analysis}

The method (Balmer profiles, Si II/III/IV ionisation equilibria, weak and strong $\mathrm{O}$ II lines) was thoroughly revised to be capable of deriving $\mathrm{T}_{\text {eff }}, \log g, \xi, \varepsilon(\mathrm{O})$ and $\varepsilon(\mathrm{Si})$ simultaneously and treating the metallicity of the underlying atmosphere in a consistent manner (for a full description see Gummersbach et al. 1998).

\section{Results for Individual Stars}

Table 1 summarizes the derived stellar parameters and abundances (in the usual notation $\varepsilon(\mathrm{X}):=\log (\mathrm{n}(\mathrm{X}) / \mathrm{n}(\mathrm{H}))+12)$. Nomenclature for cluster members is taken from Robertson (1974), for LMC field stars from Brunet et al. (1975), for SMC field stars from Azzopardi \& Vigneau (1975). Brackets around $\xi$ indicate that the microturbulence could not be co-determined simultaneously from silicon

Table 1. Derived stellar parameters

\begin{tabular}{|c|c|c|c|c|c|c|c|c|c|c|}
\hline star & $T_{\text {eff }}$ & $\log g$ & $\xi$ & $\varepsilon(\mathrm{He})$ & $\varepsilon(\mathrm{C})$ & $\varepsilon(\mathrm{N})$ & $\varepsilon(0)$ & $\varepsilon(\mathrm{Mg})$ & $\varepsilon(\mathrm{Al})$ & $\varepsilon(\mathrm{Si})$ \\
\hline BRU 217 LMC & 18100 & 2.66 & 11 & 10.80 & $\%$ & 7.60 & 8.42 & 6.90 & 5.80 & 7.12 \\
\hline BRU 231 & 17600 & 2.46 & 14 & 10.90 & $(7.80)$ & 7.90 & 8.40 & 7.00 & 5.80 & 7.05 \\
\hline NGC 1818/D1 & 24700 & 4.00 & 0 & 11.15 & 7.65 & 7.59 & 8.46 & 7.35 & 6.05 & 7.10 \\
\hline NGC $1818 / \mathrm{D} 12$ & 16850 & 2.83 & 4 & 10.90 & (8.10) & 8.00 & 8.45 & 6.75 & 5.80 & 6.90 \\
\hline NGC 2004/B15 & 19900 & 3.11 & (6) & 10.85 & 7.90 & $\%$ & 8.35 & 6.78 & 5.90 & 6.74 \\
\hline NGC 2204/B30 & 23450 & 3.34 & 14 & 10.90 & $(7.60)$ & 7.50 & 8.35 & 7.00 & 5.80 & 6.90 \\
\hline AV 175 & 19200 & 2.67 & (13) & 10.90 & $\%$ & 7.30 & 8.20 & 6.60 & 5.45 & 6.90 \\
\hline AV 218 & 23100 & 2.93 & 13 & 10.90 & 7.50 & 7.20 & 8.00 & 6.90 & 5.70 & 6.80 \\
\hline NGC 330/B30 & 16950 & 2.77 & (6) & 10.90 & (7.30) & 7.30 & 8.25 & 6.85 & $\%$ & 6.82 \\
\hline$\Delta$ (typical $)= \pm$ & 1000 & 0.2 & 2 & & & & 0.3 & & & \\
\hline
\end{tabular}




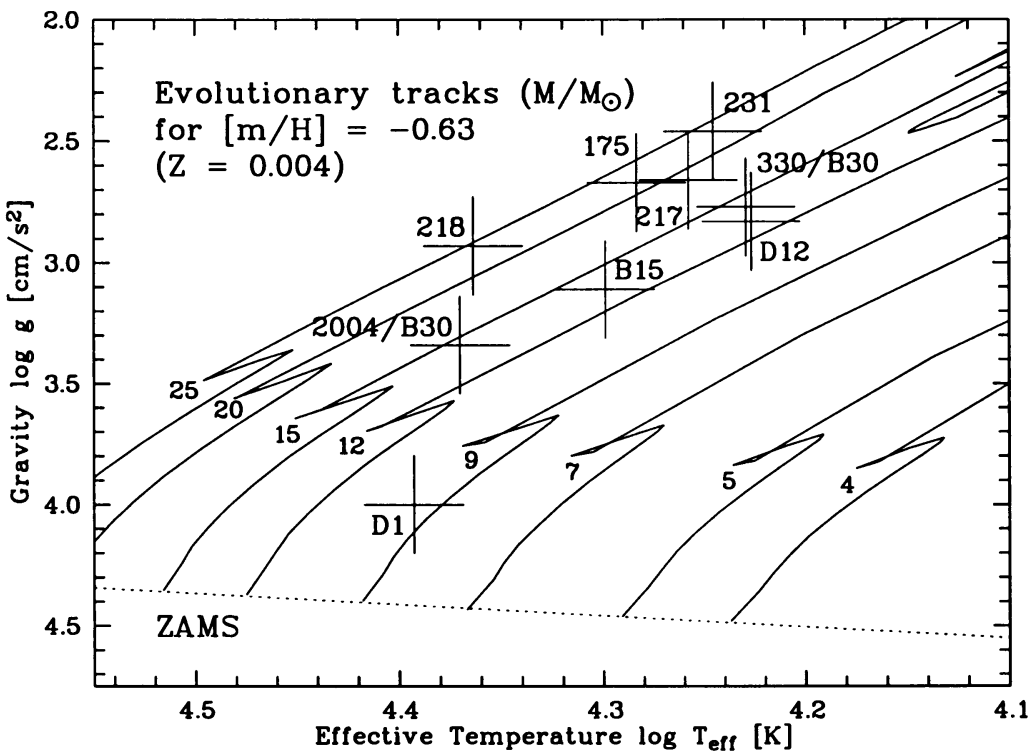

Figure 1. Kiel diagram showing the loci of the program stars with respect to evolutionary tracks according to Charbonnel et al. (1993) for a metallicity between those derived for the two Clouds.

and oxygen lines, those around $\varepsilon(C)$ that carbon is solely based on $\mathrm{C}_{\text {II }} 4267$. Compare to $\mathrm{MC} \mathrm{H}$ II data by Garnett (these proceedings)!

\section{Global Results}

- No significant metallicity difference between the clusters and their surrounding fields (but see Hill, these proceedings)

- No post-MS gap (stars on extended blue loops?)

- CNO mixing already on the MS ( $c f$. NGC 1818/D1 in Table/Figure 1), unexplained by standard evolution, possibly rotationally induced (see contributions of Langer and Venn, these proceedings).

\section{References}

Azzopardi, M., \& Vigneau, J. 1975, A\&AS, 19, 271

Brunet, J.P., et al. 1975, A\&AS, 21, 109

Charbonnel, et al. 1993, A\&AS, 101, 415

Gummersbach, C. A., et al. 1998, A\&A, 338, 881

Reitermann, A., Baschek, B., Stahl, O., \& Wolf, B. 1990, A\&A, 234, 109

Robertson, J.W. 1974, ApJ, 191, 67 